\title{
Bistum und Hochstift Würzburg im hohenlohischen Franken ${ }^{1}$
}

\author{
von Wolfgang Weiss
}

Wie aus der Themenstellung „Bistum und Hochstift Würzburg im hohenlohischen Franken" zu ersehen, zielen die Ausführungen darauf, zwei Aspekte in den Blick zu nehmen, die geistliche Herrschaft des Bischofs von Würzburg und seine weltliche als Herr des damit verbundenen Hochstifts, das zu den größten und wichtigsten geistlichen Staaten im Alten Reich zählte.

Die frühere Zugehörigkeit zum Bistum Würzburg ist vielen Bewohnern der Regionen Württembergisch oder Badisch Franken bis in die Gegenwart durchaus bewusst. Lebendige Hinweise bilden vor allem die verschiedenen Kilianspatrozinien, wie z.B. in Mulfingen, Bieringen oder Heilbronn, die als sogenannte Pertinenzpatrozinien zu deuten sind, die also durch das Patrozinium die Zugehörigkeit zur Kirche von Würzburg, als der des heiligen Kilian zum Ausdruck bringen. Kilian ist aller Franken Patron, und dieses Bewusstsein bildet eine wichtige Klammer dieser historischen Landschaft ${ }^{2}$.

Schon bei der Gründung des Bistums Würzburg durch Bonifatius 741/742 war es das Ziel, auf diese Weise die ostfränkischen Gaue kirchlich zusammenzufassen. Es ergab sich somit ein Bistumsgebiet, das sich im Norden bis zum Scheitel des Thüringer Waldes erstreckte. Im Westen gegenüber dem schon bestehenden (Erz-)Bistum Mainz bildeten Spessart und Odenwald Grenzorientierungen, wobei sich Mainz im Umfeld von Tauberbischofsheim bis vor die Tore der Stadt

1 Es handelt sich hierbei um den überarbeiteten gleichnamigen Vortrag, der am 22. Oktober 2010 im Rahmen des „6. Tag[es] der Heimatgeschichte im Hohenlohekreis“ mit dem Thema „Räder und Rechen. Zur Herrschaftsgeschichte an Kocher und Jagst im Mittelalter und Früher Neuzeit" gehalten wurde. Der Vortragsstil wurde weitgehend beibehalten; die Anmerkungen beschränken sich auf die Belege der Zitate und grundlegende weiterführende Literaturhinweise. - Allgemein sei verwiesen auf: Alfred Wendehorst: Hochstift Würzburg. In: Handbuch der Baden-Württembergischen Geschichte. Bd. 2: Die Territorien im Alten Reich. Stuttgart 1995, S. 513-525; Beschreibung des Oberamts Künzelsau (Die württembergischen Oberamtsbeschreibungen 62). Stuttgart 1881 (Nachdruck 1968); Georg Himmelheber (Bearb.): Die Kunstdenkmäler des ehemaligen Oberamts Künzelsau. Stuttgart 1962 (unveränderter Nachdruck Frankfurt am Main 1983); Der Hohenlohekreis. Amtliche Kreisbeschreibung für den Hohenlohekreis. Hg. vom Landesarchiv Baden-Württemberg in Verbindung mit dem Hohenlohekreis. 2 Bde. Ostfildern 2006.

2 Nach wie vor grundlegend: Johannes Erichsen (Hg.) unter Mitarb. v. Evamaria Brockhoff: Kilian. Mönch aus Irland - aller Franken Patron. Aufsätze (Veröffentlichungen zur Bayerischen Geschichte und Kultur 19). München 1989; Kilian. Mönch aus Irland - aller Franken Patron. 6891989. Katalog der Sonder-Ausstellung zur 1300-Jahr-Feier des Kiliansmartyriums. 1. Juli 1989-1. Oktober 1989. Würzburg 1989 (zu den Kilianspatrozinien vgl. S. 301-305). 


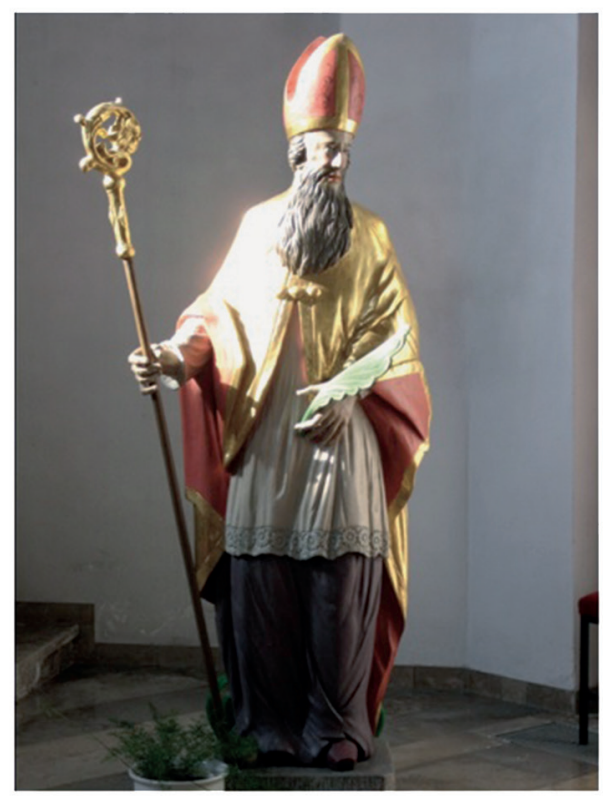

Abb. 1: St. Kilian in der Pfarrkirche Mulfingen. Foto: Wolfgang Weiß

Würzburg vorschob. Im Westen zog sich die Grenze am Neckar entlang bis nach Lauffen, von wo sie sich dann halbkreisförmig nach Murrhardt im Süden bis hin in den Raum von Fürth im Südosten und an den Obermain zog. Nach Osten hin blieb der Diözesansprengel vorläufig „offen“; in diesem Raum wurde 1007 das Bistum Bamberg gegründet.

Die Verklammerung der beiden Bistümer Mainz und Würzburg unterstreicht den starken Einfluss, den die Mainzer Kirche wenigstens im westlichen Mittelmaingebiet schon vor der Würzburger Bistumsgründung gewonnen haben muss und den sie nicht ohne Zugeständnisse aufgab. Während sich Mainz also in diesem Raum noch Gebiete sichern konnte, wurde gegenüber dem Bistum Worms, das sicher auch schon seinen Einfluss über den Neckar in Richtung Tauber vorgeschoben haben dürfte, mit der Neckarlinie eine eindeutige Abgrenzung gesucht. In den sogenannten Ausstattungsurkunden erhielt der Bischof von Würzburg 24 königliche Eigenkirchen übertragen, darunter im Südwesten die Kirchen in Lauffen, Heilbronn, Osterburken, Stöckenburg, Königshofen im Taubergau und Schwaigern. Darüber hinaus wurde Bischof Burkard der Zehnte des Ertrags von 26 Königsgütern zugesprochen, darunter von denjenigen in Heilbronn und Lauffen. Diese großzügigen Schenkungen unterstreichen das besondere Interesse der Karolinger am Bistum Würzburg. Auch wenn es historisch gesehen sicher problematisch ist, von dieser Gründungsausstattung die Brücke zum späteren bischöflichen Territorialstaat des Spätmittelalters zu schlagen, so wurden dessen 
Anfänge stets mit der Bistumsgründung verbunden und der heilige Bischof Burkard mit dem fränkischen Herzogsschwert dargestellt ${ }^{3}$.

Schon bei seiner Gründung war das Bistum Würzburg also ein politischer Faktor zur Sicherung und Umsetzung der karolingischen Herrschaft östlich des Rheins. Auch unter den Ottonen, Saliern und Staufern blieb dem Würzburger Bischof die Rolle des ersten Lehensträgers des Königs bzw. Kaisers in (Ost-)Franken. Immer wieder erhielt er durch königliche Übertragungen Rechte und Güter, die später infolge der zunehmenden Schwäche der königlichen Zentralgewalt zu einer eigenen Landesherrschaft ausgebaut werden konnten, wobei sich dabei verschiedene Konkurrenten aus dem Adel oder seitens anderer geistlicher Institutionen zeigten, im Südwesten des Bistums die aufstrebenden Hohenloher, die es nicht zuletzt als Würzburger Lehensempfänger zu Macht und Ansehen brachten, sowie das Erzstift Mainz.

Wenden wir uns aber zuerst weiter dem Aspekt Bistum und damit der kirchlichen Seite zu. Bis ins 12. Jahrhundert hatte sich die Zahl der Kirchen so verdichtet, dass zu deren Verwaltung und Kontrolle im weiten Bistum Würzburg Untergliederungen, und zwar Archidiakonate, eingerichtet wurden. Spätere Aufzeichnungen nennen zwölf Archidiakonate. Der Archidiakon hatte in seinem Sprengel die bischöflichen Gerichts- und Aufsichtsrechte wahrzunehmen. Dies geschah in erster Linie durch die jährliche Abhaltung des Sendgerichtes (Send = Synode). Jedem Archidiakonat war ein Landkapitel zugeordnet, in dem - anfangs wohl unter dem Vorsitz des Archidiakons - die Landgeistlichen zusammengeschlossen waren. Aus ihrer Mitte wählten sie den Dekan, der die Kapitelsgeistlichkeit (z. B. bei der Diözesansynode) repräsentierte ${ }^{4}$.

Später wurden einige Archidiakonate wegen ihrer Größe in mehrere Landkapitel aufgeteilt. Für unseren Raum ist das Archidiakonat VI von Bedeutung, das die Pfarreien im Jagst- und Kochergebiet umfasste und sich im Spätmittelalter in

3 Vgl. Jürgen Lenssen, Ludwig Wamser (Hg.): 1250 Jahre Bistum Würzburg. Archäologisch-historische Zeugnisse der Frühzeit. Würzburg 1992 (besonders Erik Soder von Güldenstubbe: Bistum und Hochstift Würzburg. Zwei Begriffe, zwei verschiedene Inhalte, S. 11-30, und Heinrich Wagner: Die Zehntenschenkung Pippins für Würzburg [751/2], S. 35-38); vgl. Wolfgang Weiß: Linien der Missionierungs- und Christianisierungsgeschichte Frankens. In: Rolf Bergmann u. a. (Hg.): Missionierung und Christianisierung im Regnitz- und Obermaingebiet (Historischer Verein Bamberg, Schriftenreihe 41). Bamberg 2007, S. 119-136 (mit zahlreichen weiterführenden Literaturhinweisen).

4 Vgl. Erik Soder von Güldenstubbe: Die Entwicklung der kirchlichen Strukturen im Bistum Würzburg. In: Peter Kolb, Ernst-Günter Krenig (Hg.): Unterfränkische Geschichte. Bd. 2: Vom hohen Mittelalter bis zum Beginn des konfessionellen Zeitalters. Würzburg 1992, S. 215-232; vgl. Julius Krieg: Der Kampf der Bischöfe gegen die Archidiakone im Bistum Würzburg (Kirchenrechtliche Abhandlungen 12). Stuttgart 1914; ders.: Die Landkapitel im Bistum Würzburg bis zum Ende des 14. Jahrhunderts (Görres-Gesellschaft, Veröffentlichungen der Sektion für Rechts- und Sozialwissenschaft 28). Paderborn 1916; ders.: Die Landkapitel im Bistum Würzburg von der zweiten Hälfte des 14. bis zur zweiten Hälfte des 16. Jahrhunderts (Kirchenrechtliche Abhandlungen 99). Stuttgart 1923; Franz J. Bendel: Quellenbeiträge zum mittelalterlichen Send im Bistum Würzburg. In: Würzburger Diözesangeschichtsblätter 6 (1938), S. 1-20. 
drei Landkapitel, und zwar Crailsheim, (Schwäbisch) Hall und eben auch Künzelsau, aufteilte. Nach der Diözesanmatrikel aus der Mitte des 15. Jahrhunderts gab es im Landkapitel Künzelsau 48 Orte, in denen sich über 60 Pfarreien und Benefizien befanden 5 . 1487 verlor allerdings der Ganerbenort - ein Ort mit mehreren Besitzern, die aber gemeinschaftlich die Herrschaft ausübten - Künzelsau seine Funktion als Sitz des Landkapitels. Er wurde in das hohenlohische Ingelfingen verlegt. Der Grund wird in der Rivalität der Herren von Stetten und der Hohenloher zu suchen sein ${ }^{6}$.

In der Mitte des 16. Jahrhunderts fand infolge der Reformation die Kapitelsorganisation an Kocher und Jagst ihr vorläufiges Ende. Letztmals trat das Landkapitel Ingelfingen 1545 zusammen. Als in den 1580er Jahren die Würzburger Diözesanleitung unter dem tatkräftigen Bischof Julius Echter wieder versuchte, das Kapitel zu beleben, kamen gerade noch 11 Geistliche (von ursprünglich 45 Pfarrstellen und 23 weiteren Benefizien) zusammen, und zwar der Pfarrer von Mulfingen und der dortige Frühmesser sowie die Pfarrer von Ailringen, Amrichshausen, Sindeldorf, Krautheim, Oberginsbach, Assamstadt, Marlach, Rengershausen und der Kaplan von Klepsau, also Geistliche von Orten des Erzstifts Mainz, des Deutschen Ordens sowie des Hochstifts Würzburg und damit des Amts Jagstberg, auf das ich in den weiteren Schritten wiederholt noch näher eingehen werde ${ }^{7}$. In den übrigen Orten hatte man sich der Reformation zugewandt. Da in reichsständischen Territorien gemäß Augsburger Religionsfrieden von 1555 die Landesherrn das ius reformandi und damit das Recht erhalten hatten, für ihre Untertanen die Konfession zu bestimmen, und bei seiner Entscheidung für die Confessio Augustana, also das lutherische Bekenntnis, auch die jeweiligen Diözesanrechte suspendiert waren, besaß der Würzburger Bischof nur noch über Geistliche katholischer Territorien die Jurisdiktion und eine Zugriffsmöglichkeit ${ }^{8}$.

Erst 1591 konnte das Landkapitel wieder, allerdings nicht mehr im hohenlohischen und damit evangelischen Ingelfingen, sondern im Mainzischen Krautheim gegründet werden. 1594 fand im Landkapitel Krautheim auch eine Visitation

5 Franz J. Bendel: Die Würzburger Diözesanmatrikel aus der Mitte des 15. Jahrhunderts. In: Würzburger Diözesangeschichtsblätter 2, Heft 2 (1934), S. 1-46 („Capitulum Kunczeslaw“ S. 10); zu den kirchlichen Verhältnissen in Künzelsau vgl. Elizabeth Wainwright: Studien zum deutschen Prozessionsspiel. Die Tradition der Fronleichnamsspiele in Künzelsau und Freiburg und ihre textliche Entwicklung (Münchener Beiträge zur Mediävistik und Renaissance-Forschung 16). München 1974, S. 45-50.

6 Vgl. Werner Nowak: Die Ganerbschaft Künzelsau. Geschichte, Organisation, Bedeutung. Plochingen am Neckar 1966, S. 31-35.

7 Vgl. Helmut Neumaier: Reformation und Gegenreformation im Bauland (FWrF 13). Schwäbisch Hall 1977, S. 212 f.; vgl. allgemein J[ohann] Scheffold: Zur Geschichte des Landkapitels Amrichshausen. Heilbronn 1882.

8 Vgl. Der Hohenlohekreis. Bd. 1 (wie Anm. 1), S. 213-224; vgl. Peter Schiffer: Die Entwicklung der konfessionellen Landkarte. In: Ders. (Hg.): Aufbruch in die Neuzeit. Das nördliche Württemberg im 16. Jahrhundert (FWrF 53). Ostfildern 2012, S. 69-80. 
durch Beauftragte des Bischofs von Würzburg statt. Die Visitationen hatten in erster Linie die Aufgabe, Geistliche und Kirchenvolk, die weithin in einem vorkonfessionellen Status mit Tendenzen zu reformatorischen Gebräuchen verharrten, zu einem eindeutig katholischen Profil zu führen?.
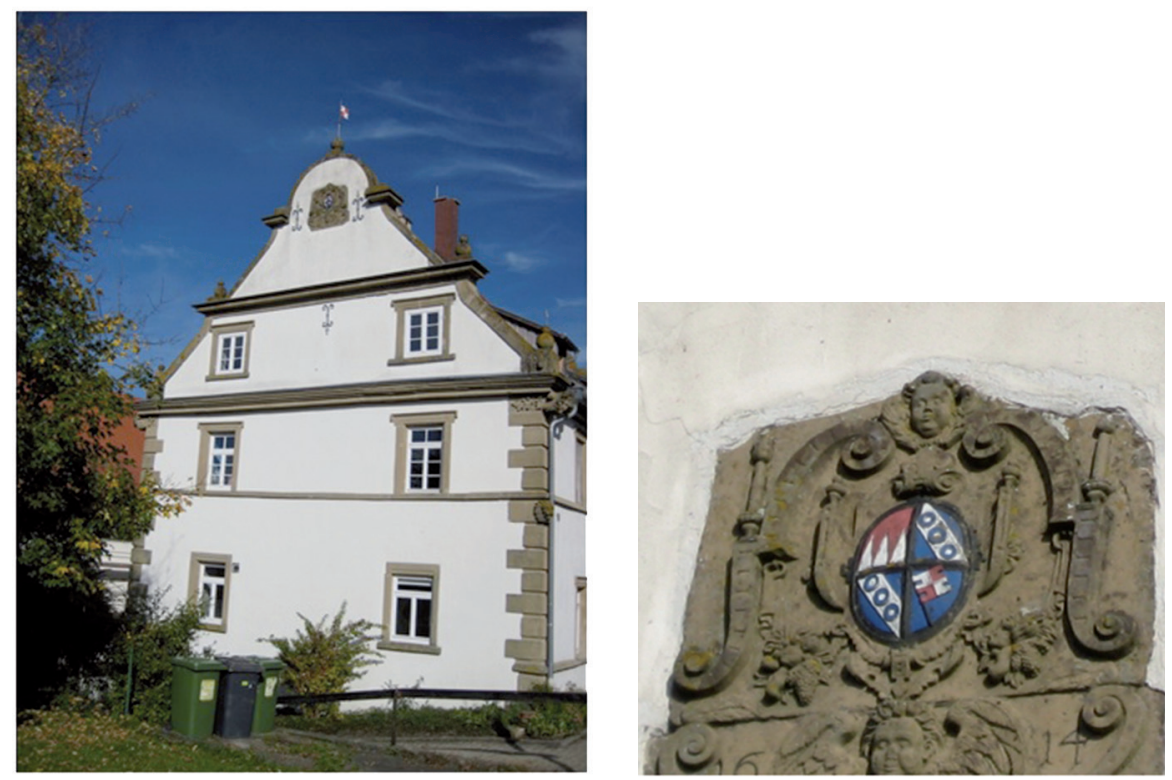

Abb. 2 und 3: Amtsgebäude im Schloss Jagstberg mit Echterwappen im Giebel. Fotos: Wolfgang Weiß

Besondere Einwirkungsmöglichkeiten hatte der Würzburger Bischof, wo er unmittelbar Landesherr war ${ }^{10}$. So wurde Julius Echter in seinem Amt Jagstberg auf verschiedene Weise im Dienste des Ausbaus der Landesherrschaft und der katholischen Restauration aktiv. Zum einen baute er den Amtssitz Schloss Jagstberg aus, indem er 1582 ein neues Kellereigebäude und 1605/1606 ein stattliches Wohngebäude für den Amtmann bzw. Amtsverweser errichten ließ ${ }^{11} .1597$ grün-

9 Vgl. Neumaier (wie Anm. 7), S. 214-221.

$10 \mathrm{Zu}$ den territorialen Verhältnissen: vgl. Karl Schumm: Aus der Geschichte des Kreisgebietes. In: Konrad Theiss, Hermann Baumgartner (Hg.): Der Kreis Künzelsau. Aalen/Stuttgart 1965, S. 98 159 (bes. S. 127 f.); vgl. Der Hohenlohekreis. Bd. 1 (wie Anm. 1), S. 35-57.

11 Vgl. Wilhelm Engel: Eine Finanzstatistik über die Bauten des Fürstbischofs Julius Echter. In: Max H. von Freeden, Wilhelm Engel: Fürstbischof Julius als Bauherr (Mainfränkische Hefte 9). Würzburg 1951, S. 62-105 (bes. S. 83 f., Nr. 211-213); zur Bautätigkeit Echters in dieser Gegend vgl. Wolfgang Müller: Beobachtungen zum Bau der Dorfkirchen zur Zeit des Bischofs Julius Echter von Mespelbrunn. In: Würzburger Diözesangeschichtsblätter 35/36 (1974), S. 331-347; Hermann Hofmann: Vier Archivalien (1611-1614) über Julius-Echter-Bauten außerhalb Würzburgs. In: Würzburger Diözesangeschichtsblätter 37/38 (1975), S. 687-745 (bes. S. 694). 


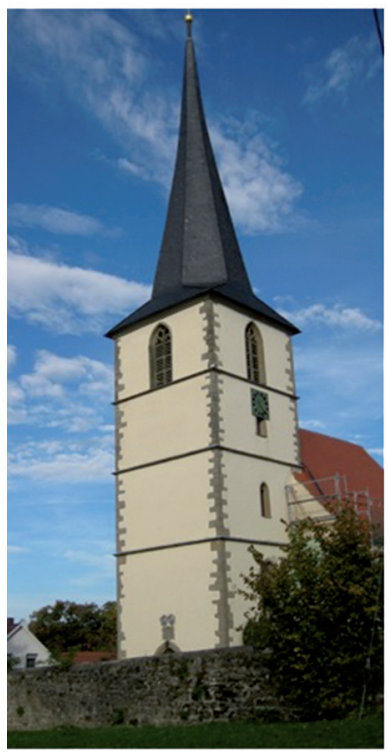

Abb. 4: Echterturm Amrichshausen. Foto: Wolfgang Weiß

dete er die Pfarrei Jagstberg ${ }^{12}$, bis dahin nur eine Burg- bzw. Schlosskaplanei, und baute hier eine neue Kirche. Schon die Kapelle trug das relativ seltene Patrozinium des heiligen Burkard. Dies wird den propagandistischen Absichten Julius Echters durchaus entgegengekommen sein, verwies es doch auf den ersten Würzburger Bischof und im Verständnis der Zeit ersten geistlichen Herzog von Franken. Es dokumentierte damit auch seinen Anspruch auf diesen Raum. In Amrichshausen ließ er eine neue Kirche erbauen, die geradezu als Musterbeispiel einer Echterkirche betrachtet werden kann. Vor allem der hochgezogene Echterturm sollte ein sinnenfälliges Zeichen für den wiedererstarkenden Katholizismus in diesem Raum sein und so etwas wie ein katholisches Ausrufezeichen in eine evangelisch dominierte Landschaft hinein ${ }^{13}$. Wie auch sonst im Hochstift hat Echter seine Untertanen im Amt Jagstberg vor die Wahl gestellt, sich zur katholischen Konfession zu bekennen oder auszuwandern. Dass der Katholizismus sich aber nicht nur durch äußere Maßnahmen stabilisieren ließ, war Echter ebenso klar. Es musste wieder eine mentale Bindung an katholische Frömmigkeitsformen wachsen. Hier spielte sicher die Wiederbelebung des Annafestes in

12 Vgl. Alfred Wendehorst (Bearb.): Das Bistum Würzburg. Teil 3: Die Bischofsreihe von 1455 bis 1617 (Germania sacra NF 13). Berlin/New York 1978, S. 206.

13 Vgl. Himmelheber (wie Anm. 1), S. 73-81; vgl. auch das populäre Werk von Carheinz Gräter, Jörg Lusin: Kirchen, Klöster und Kapellen in Hohenlohe. Geschichte und Geschichten. Tübingen 2007, S. 3 f. 
Mulfingen eine Rolle. In diesem Sinne war das Wunderjahr 1597 eine eindrucksvolle Botschaft, dass der katholische Glaube sich als der wirkmächtigere erwies $^{14}$. Ohne diese Maßnahmen im Detail zu bewerten - aus heutiger Sicht ist hier sicher manches als problematisch zu betrachten -, ist aber unbestritten, dass es Julius Echter und seinen Nachfolgern als Würzburger Bischöfe gelang, dem Amt Jagstberg eine katholische Identität zu geben, die nicht nur in der geistlichen Topographie bis heute erkennbar ist.

Im frühen 17. Jahrhundert bildeten die schon genannten Pfarreien mit den Pfarreien Gommersdorf (erst 1598 mit Pfarrrechten ausgestattet ${ }^{15}$ ) und Westernhausen (mit Winzenhofen) sowie den kläglichen Resten des Haller und Crailsheimer Kapitels, vornehmlich die zum Bistum Würzburg gehörigen Pfarreien in der Fürstpropstei Ellwangen, und zwar Bühlertann, Bühlerzell, Hohenberg, Jagstzell und Stimpfach, das Landkapitel Krautheim ${ }^{16}$. Letztere Orte wurden im 18. Jahrhundert mit den Comburgischen Orten Steinbach, Allmerspann und Hausen an der Roth sowie (Markt-)Lustenau im relativ kleinen Landkapitel Bühlertann ${ }^{17}$ zusammengefasst. Zur Information sei noch darauf hingewiesen, dass Schöntal, Berlichingen und Bieringen zum Würzburger Landkapitel Buchen gehörten ${ }^{18}$. In den folgenden Jahrzehnten erlebte das Landkapitel Krautheim keine größeren Veränderungen. Nur eine einzige weitere Pfarrei kam dazu, nämlich im mainzischen Gebiet der Ort Nagelsberg, ursprünglich eine Filiale von Künzelsau, dann vorübergehend Amrichshausen unterstellt ${ }^{19}$. Julius Echter besaß als Ganerbe von Künzelsau ab 1583 Ambitionen, auch hier den alten Glauben wieder aufzurichten. Er fand auch die Unterstützung eines weiteren Ganerben, nämlich Mainz. Hohenlohe und Stetten widersetzten sich aber. Die katholische Seite konnte sich nicht durchsetzen, weil die Hohenloher die Schutzherren der Künzelsauer Kirche waren. 1662 kauften die Hohenloher dem Stift Comburg die Patronatsrechte der Künzelsauer St. Johannis-Pfarrei ab und konnten so das evangelische Bekenntnis dauerhaft sichern ${ }^{20}$.

In späterer Zeit traten dazu noch die Kuratien im Gebiet der 1667 zur katholischen Konfession konvertierten Hohenloher (und zwar die Linien Schillingsfürst und Bartenstein). Es gab somit im 18. Jahrhundert wieder katholische Seelsorgestellen in Waldenburg, Pfedelbach, Kupferzell und Bartenstein. Darüber

14 Vgl. Jürgen Hermann Rauser: Mulfinger Heimatbuch (Heimatbücherei Hohenlohekreis 1). Künzelsau 1980, S. 388-398; Himmelheber (wie Anm. 1), S. 230 f.; Gräter, Lusin (wie Anm. 13), S. 88-90.

15 Vgl. Der Hohenlohekreis. Bd. 1 (wie Anm. 1), S. 425 f.

16 Vgl. Hans Eugen Specker: Die Landkapitel im Bistum Würzburg zu Beginn des 17. Jahrhunderts. In: Würzburger Diözesangeschichtsblätter 24 (1962), S. 285-292 (bes. S. 289).

17 Vgl. Winfried Romberg: Das Würzburger Pfarrwesen vom Dreißigjährigen Krieg bis zur Säkularisation (1617-1803). Institutionen und Pastoral im Spiegel der landesherrlichen Kirchenordnungen. In: Würzburger Diözesangeschichtsblätter 73 (2011), S. 95-158 (bes. S. 154).

18 Vgl. Specker (wie Anm. 16), S. 289.

19 Vgl. Himmelheber (wie Anm. 1), S. 234 f.

20 Vgl. Nowak (wie Anm. 6), S. 118-122. 
hinaus ist noch Meßbach, die Residenz der katholischen Freiherrn von Eyb, zu nennen ${ }^{21}$. Eine Rückführung der jeweiligen Pfarreien zur katholischen Konfession war aber reichsrechtlich nicht möglich, weil der Westfälische Frieden der jeweiligen Konfession ihren Besitzstand des Normaljahres 1624 garantierte. Insgesamt lässt sich festhalten, dass die Jurisdiktion des Bischofs von Würzburg mit der Reformation im hohenlohischen Franken als nunmehr mehrheitlich evangelischem Gebiet auf dann doch relativ wenige Orte des Mainzer Amtes Krautheim, der Schöntaler Klosterorte, des Deutschen Ordens und seines unter seiner weltlichen Herrschaft stehenden Amtes Jagstberg begrenzt war.

Dieses doch relativ überschaubare Amt Jagstberg ${ }^{22}$ bildete zusammen mit dem Würzburger Anteil im Ganerbenort Künzelsau den südwestlichsten Teil des Hochstifts Würzburg, sieht man einmal von der relativ selbstständigen Würzburger Mediatherrschaft Ritterstift Comburg und ihren Orten sowie dem Amt Braunsbach des Würzburger Domkapitels ab. Das Amt Jagstberg bestand nach dem in der Würzburger Universitätsbibliothek verwahrten „Verzeichnis des Hochstifts Würzburg aus dem Jahr $1699^{“ 23}$ aus Schloss, Stadt und Pfarrei Jagstberg mit 44 Bürgern, also selbstständigen Haushaltungen, sowie den Pfarrdörfern Amrichshausen mit 27 Untertanen (wiederum Haushaltsvorständen) und Mulfingen, der größte Ort mit 128 Untertanen. Etwas diffus bleibt die Zuweisung von Simmetshausen, Alkertshausen und Mäusberg, die unmittelbar unter der Rubrik Mulfingen aufgeführt werden. Dann werden genannt die Dörfer Simprechtshausen mit 45 Untertanen und Zaisenhausen mit 47 Untertanen - damals noch nicht als eigene Pfarreien, sondern Filialen von Mulfingen, - sowie eine Reihe von Weilern, nämlich Hohenroth mit 13 Untertanen, Ochsental mit 7 Untertanen und Seidelklingen mit 13 Untertanen. Als Orte mit Würzburger Untertanen und Besitzungen werden noch Heimhausen und Steinbach erwähnt. Zaisenhausen war bis 1666 ein Kondominatsort des Hochstifts Würzburg und der Grafen von Hohenlohe-Schillingsfürst. In diesem Jahr kaufte das Hochstift Würzburg die im Besitz der Grafen von Hohenlohe-Schillingsfürst befindliche Hälfte für 9600 Gulden. Solche Aktivitäten waren im Sinne der Vereinheitlichung und Arrondierung des Territoriums sowie der Ausbildung einer modernen Staatlichkeit durchaus charakteristisch für die frühe Neuzeit. Nicht selten wurden dafür auch Tauschaktionen durchgeführt.

21 Vgl. Würzburger Hof- und Staatskalender für die Jahre 1747-1802 (jeweils mit Aufstellung der Landkapitel mit ihren Seelsorgestellen und Geistlichen); vgl. Der Hohenlohekreis. Bd. 1 (wie Anm. 1), S. 221-224.

22 Vgl. Erwin Riedenauer: Die Landämter des Hochstifts Würzburg und ihr Personal im 17. und 18. Jahrhundert. In: Würzburger Diözesangeschichtsblätter 37/38 (1975), S. 339-465.

23 Edition dieses Verzeichnisses: Alfred Schröcker (Bearb.): Statistik des Hochstifts Würzburg um 1700 (Quellen und Forschungen zur Geschichte des Bistums und Hochstifts Würzburg 30). Würzburg 1977, Beschreibung Amt Jagstberg (S. 102f.), Stift Komburg (S.13f .), Ganerbenort Künzelsau (S. 62-65). 
Mit der Herrschaft Jagstberg war noch die Zent, also die Hochgerichtsbarkeit verbunden, und zwar neben den genannten Amtsdörfern noch im hohenlohischen Dorf Hohebach, im zu Kloster Schöntal gehörigen Weldingsfelden sowie im Deutschordensort Ailringen „bis an den Bach“"24. Die Amtsbeschreibung macht deutlich, wie wenig wir es in diesem Raum mit geschlossenen Herrschaften zu tun haben und wie einzelne Hoheitsrechte sich überlappen. Man spricht daher bezüglich der fränkischen Situation von territoria non clausa, von nicht geschlossenen Territorien.

Die Amtsbeschreibung des Jahres 1699 spricht davon, dass die Burg Jagstberg von den Templern erbaut worden sei und bei deren Auflösung 1310 an das Hochstift Würzburg gelangt sei ${ }^{25}$. Urkundlich ist dagegen belegt, dass der Würzburger Bischof Berthold II. von Sternberg 1275 von Albrecht von Ebersberg die Lehenshoheit über die Burg Jagstberg erwarb ${ }^{26}$. Mit der Lehenshoheit war aber nicht die unmittelbare Nutzung der Burg sowie ihrer Güter und Einnahmen verbunden. Diese lag als Würzburger Lehen in den Händen der Hohenlohe-Brauneck. Diese verkauften sie 1340 an Pfalz-Bayern bzw. an die Wittelsbacher ${ }^{27}$, während sie von diesen wiederum 1387 der Würzburger Bischof Gerhard von Schwarzburg erwarb, zunächst für das Haus Schwarzburg, dann aber für das Hochstift ${ }^{28} .1388$ hatte aber das Stift wieder Finanzbedarf und musste die gerade erworbene Stadt und Burg Jagstberg für 2000 Gulden verpfänden ${ }^{29}$. Aus dem Jahr 1406 ist wiederum überliefert, dass der Würzburger Bischof Johann von Egloffstein Ansprüche auf Jagstberg und Lauda von Johann von Hohenlohe(-Speckfeld) kaufte ${ }^{30}$, aus dem Jahr 1410, dass der gleiche Bischof wieder Burg, Stadt und Amt für 3400 Gulden verpfändete ${ }^{31}$. Aus dem Jahr 1428 ist bekannt, dass der Würzburger Bischof Johann von Brunn sie den Hornbergern als Pfandlehen übergab. Mit Horneck von Hornberg, einem Meister der gewinnbringenden Fehde andere nennen ihn einen Raubritter - entspann sich in den späten 1430er Jahren ein länger dauernder Konflikt. Die Windungen und Wendungen dieser Auseinandersetzung können hier nicht entfaltet werden. Die Burg wurde mehrfach belagert, hin und her erobert. Schließlich übergab der Stiftspfleger Johann von Limpurg im Jahre 1444 dem Hans von Absberg die Burg als Pfandlehen ${ }^{32}$.

24 Ebd., S. $102 \mathrm{f}$.

25 Vgl. ebd., S. 102.

26 Vgl. Alfred Wendehorst: Das Bistum Würzburg. Teil 2: Die Bischofsreihe von 1254 bis 1455 (Germania sacra NF 4). Göttingen 1969, S. 24 (bei Wendehorst jeweils die genauen Urkundenbelege).

27 Vgl. Gerhard Kölbel: Historisches Lexikon der Deutschen Länder. Die deutschen Territorien vom Mittelalter bis zur Gegenwart. München ${ }^{2} 1989$, S. 555; vgl. Rauser (wie Anm. 14), S. 305-308 (zum Teil in Anlehnung an die Beschreibung des Oberamts Künzelsau).

28 Vgl. Wendehorst, Würzburg 2 (wie Anm. 26), S. 112.

29 Vgl. ebd., S. 117.

30 Vgl. ebd., S. 134.

31 Vgl. ebd., S. 136.

32 Vgl. Beschreibung des Oberamts Künzelsau (wie Anm. 1), S. 586f.; Wendehorst, Würzburg 
Die Vorgänge belegen einerseits die schwierigen, ständig fluktuierenden, ja geradezu chaotischen Herrschaftsverhältnisse im Spätmittelalter und die immensen finanziellen Schwierigkeiten des Hochstifts in dieser Periode, die vor allem aus den ständigen Streitigkeiten des Bischofs mit dem Domkapitel und auch den hochstiftischen Städten resultierten und die das Hochstift an den Rand des Ruins führten. Seitens des Würzburger Domkapitels wurde 1441 sogar überlegt, das Hochstift an den Deutschen Orden zu verkaufen ${ }^{33}$.

Unter Fürstbischof Rudolf von Scherenberg stabilisierte sich aber das Hochstift in der zweiten Hälfte des 15. Jahrhunderts wieder organisatorisch und finanziell. Der Fürstbischof konnte Schulden tilgen und Mittel zur Auslösung verpfändeter Ämter und Orte bereitstellen. Er schuf so die Voraussetzung für den Würzburger Territorialstaat der frühen Neuzeit. Schloss und Amt Jagstberg löste Fürstbischof Scherenberg 1475 aus $^{34}$. Von nun stand das Amt in bischöflicher Eigenverwaltung durch einen zeitweiligen Amtmann bzw. Amtsverweser. Für das lebendige Interesse an diesem Gebiet spricht auch, dass Bischof Lorenz von Bibra 1496 der Stadt Jagstberg Siegel und Wappen ${ }^{35}$ verlieh und 1499 einen Anteil an der Ganerbschaft Künzelsau von den Herren von Stetten ${ }^{36}$ erwarb. Zu Beginn des 16. Jahrhunderts gelang es auch, das immer noch vorhandene Wiedereinlösungsrecht der Wittelsbacher (aus dem Erwerb von 1387) zu beseitigen ${ }^{37}$. Insgesamt zeigt sich, dass die Würzburger Bischöfe ihre Präsenz in diesem Raum verteidigen und das Amt Jagstberg als eine Art territorialen Brückenkopf im Südwesten des Bistums bzw. Hochstifts sichern wollten.

Wer die mittelalterliche Entwicklung betrachtet, wird sogar feststellen, dass die Würzburger ursprünglich größere Ambitionen in diesem Raum hatten. So gehörte ihnen in der zweiten Hälfte des 14. Jahrhunderts die Hälfte von Burg und Stadt Krautheim, mussten sie aber dann wegen der angesprochenen Konflikte im Kernbereich des Hochstifts dem Erzstift Mainz überlassen ${ }^{38}$. Für die frühere Bedeutung in diesem Raum spricht auch die Würzburger Lehensherrschaft über zahlreiche Hohenloher Besitzungen wie Buchenbach, Krailshausen, Langenburg, Weikersheim, Wilhermsdorf und Neidhardswinden, Schillingsfürst und

2 (wie Anm. 26), S. 181; Lorenz Fries: Chronik der Bischöfe von Würzburg 742-1495. Bd. VI: Die Miniaturen der Bischofschronik (Fontes Herbipolenses VI). Würzburg 1996. S. 183 (Miniatur Nr. 156: Eroberung von Schloß Jagstberg, 1445); Hermann Ehmer: Horneck von Hornberg. Raubritter oder Opfer fürstlicher Politik? In: Kurt Andermann (Hg.): „Raubritter“ oder „Rechtschaffene vom Adel"? Aspekte von Politik, Friede und Recht im späten Mittelalter (Oberrheinische Studien 14). Sigmaringen 1997, S. 65-88.

33 Vgl. Wendehorst, Würzburg 2 (wie Anm. 26), S. 167.

34 Vgl. Sebastian Zeissner: Rudolf II. von Scherenberg. Fürstbischof von Würzburg 1466-1495. Würzburg ${ }^{2} 1952$, S. $36 \mathrm{ff}$.

35 Vgl. Wendehorst, Würzburg 3 (wie Anm. 12), S. 61.

36 Vgl. Nowak (wie Anm. 6), S. 91 f.

37 Vgl. Wendehorst, Würzburg 3 (wie Anm. 12), S. 58.

38 Vgl. Theodor Humpert: Die territoriale Entwicklung von Kurmainz zwischen Main und Neckar. Würzburg 1913, S. 53. 
Bartenstein. Es erfolgte bis zum Ende des Alten Reiches die Belehnung der Hohenlohe durch den Würzburger Bischof ${ }^{39}$. Die Vasallität war aber an sich ohne große faktische Bedeutung. Die Lehen wurden nämlich vererbt. Es gab Leihezwang, und nur beim völligen Aussterben eines Geschlechts in der männlichen Linie (Mannlehen) bot das Heimfallrecht die Möglichkeit, die Territorialherrschaft auszubauen, wie dies dem Bistum Würzburg z.B. bei den Wertheimern oder Hennebergern gelang. Die Lehen stammen aus dem Hochmittelalter, als die Hohenlohe zur engeren Umgebung der Würzburger Bischöfe gehörten. Seit dem 12. Jahrhundert sind sie Afterlehensträger des Würzburger Bischofs. Das angesprochene Lehensverhältnis spiegelt aber wider, wie sich die Tradition der personellen Bindung im sogenannten Personenverbandsstaat und das modernere Prinzip der Territorialität nicht einfach ablösten, sondern bis ins 19. Jahrhundert fortlebten. Weitere Würzburger Vasallen waren noch die Adelsheim, Berlichingen, Eyb und Stetten ${ }^{40}$.

Am Ende des alten Reiches zählt der Hof- und Staatskalender von 1801 bezüglich Jagstberg sieben Amtsortschaften mit Schultheissen und Bürgermeister auf, und zwar Amrichshausen, Hohenroth, Mulfingen, Ochsental, Seidelklingen, Simprechtshausen und Zaisenhausen. In der Stadt Jagstberg gibt es einen Oberund Unterbürgermeister. Zu diesem Zeitpunkt befindet sich allerdings der Amtssitz nicht mehr in Jagstberg, sondern seit 1781/82 im größten Amtsort, nämlich Mulfingen. Noch heute schmückt das Wappen des damaligen Bischofs Franz Ludwig von Erthal das vormalige Amtsgebäude und heutige Rathaus von Mulfingen ${ }^{41}$. Letzter bürgerlicher Amtsverweser zu Jagstberg und Künzelsau - daneben gab es noch den adeligen Oberamtmann Franz Gottlieb Freiherr von Guttenberg, der zugleich im Amt Haltenbergstetten und Laudenbach als Oberamtmann fungierte - war 1801 ein Philipp Peter Fischer, zugleich Zentgraf (Vorsitzender des Zentgerichts) und Forstmeister. An weiteren Beamten gab es den Amts- und Zent-Physicus Dr. phil. und Dr. med. Georg Daniel Wiebel, daneben den nichtakademischen Amts- und Zentchirurgus Franz Mensch. In Mulfingen wirkten noch der Amts-, Gegen-, Zent- und Zunftschreiber, Oberaccisor und Guldenszöllner Eduard Götz sowie der Zollbereiter Mathäus Gatz (gleichzeitig auch noch Revierjäger). In Künzelsau ${ }^{42}$ waren tätig der Ganerbschaftliche Schultheiß Georg Friedrich Fischer und der Ganerbschaftliche Amts- und Gerichtsschreiber Friedrich Carl Fischer ${ }^{43}$.

39 Vgl. Gerhard Taddey: Aus der Geschichte der Lehnsbeziehungen zwischen Würzburg und Hohenlohe. In: Würzburger Diözesangeschichteblätter 42 (1980), S. 235-243; vgl. Wilhelm Engel: Würzburg und Hohenlohe. Zwei Untersuchungen zur Fränkischen Geschichte des hohen und späten Mittelalters (Mainfränkische Hefte 2). Würzburg 1949.

40 Die Vasallen werden auch im Würzburger Hof- und Staatskalender (z. B. 1801, S. 111-114) immer detailliert aufgelistet; vgl. auch Schröcker (wie Anm. 23), S. 26-30.

41 Vgl. Himmelheber (wie Anm. 1), S. 232; Rauser (wie Anm. 14), S. 366 und 427.

42 Fraglich muss bleiben, ob er zu diesem Zeitpunkt noch vom 1710 erbauten, repräsentativen

Würzburger Amtshaus aus seine Aufgaben erfüllte; vgl. Himmelheber (wie Anm. 1), S. 56.

43 Vgl. Würzburger Hof- und Staatskalender für das Jahr 1801, S. 139 und $146 \mathrm{f}$. 


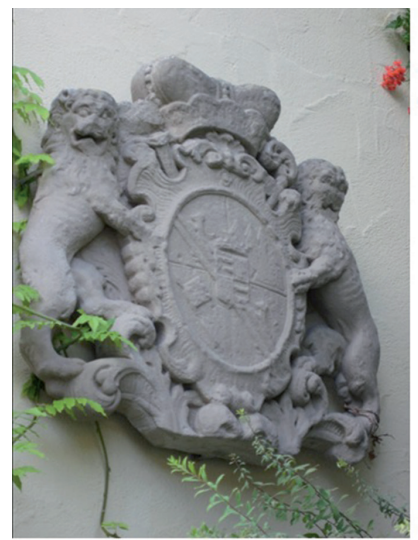

Abb. 5: Wappen des Fürstbischofs Franz Ludwig von Erthal (1779-1795) am heutigen Rathaus in Mulfingen. Foto: Wolfgang Weiß

Mit der Säkularisation 1802/03 endete die Zeit der weltlichen Herrschaft des Würzburger Bischofs an Jagst und Kocher. Amrichshausen und der Würzburger Anteil von Künzelsau gingen an Hohenlohe-Oehringen (bzw. Neuenstein), der Hauptteil des Amtes wurde dem Prinzen Karl-Joseph von Hohenlohe-Bartenstein, der sich nun von Hohenlohe-Jagstberg nannte, zugeschlagen. Aber schon 1806 erfolgte die Mediatisierung der Hohenloher Linien; ihre Gebiete wurden Teil des neuen Königreichs Württemberg (teilweise auch des Königreichs Bayern). Bald nahm auch die Zeit des Bistums Würzburg in diesem Raum ihr Ende: 1812 standen die Katholiken (vorerst provisorisch) unter der geistlichen Administration des für die württembergischen katholischen Pfarreien errichteten Generalvikariats Ellwangen. 1817 wurde das Württembergische Generalvikariat nach Rottenburg verlegt. Die Errichtung des Bistums Rottenburg erfolgte $1821 / 1827$.

Die Zeit des Würzburger Rechens in diesem Raum war nun vorbei. Das Wappen des alten Landkreises Künzelsau hat noch an die frühere Würzburger Zeit erinnert; im größeren Hohenlohe-Kreis war dann der Würzburger Anteil zu marginal, um im Landkreiswappen eigens Berücksichtigung zu finden. Vergessen ist die Würzburger Zeit in geistlicher und weltlicher Hinsicht aber nicht. Ein Stück Erinnerungskultur wollen diese Ausführungen leisten.

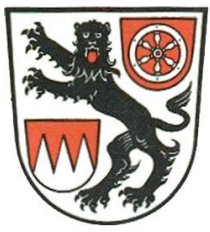

Abb. 6: Wappen des ehemaligen Landkreises Künzelsau. 